\title{
How do different lighting conditions affect the vision and quality of life of people with glaucoma? A systematic review
}

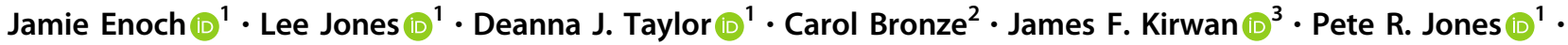 \\ David P. Crabb $\mathbb{B}^{1}$
}

Received: 30 September 2019 / Revised: 15 October 2019 / Accepted: 21 October 2019 / Published online: 10 December 2019

(c) The Author(s), under exclusive licence to The Royal College of Ophthalmologists 2019

\begin{abstract}
This article is a systematic review of evidence regarding the impact of different lighting conditions on the vision and quality of life $(\mathrm{QoL})$ of people with primary open-angle glaucoma (POAG). A systematic literature search was carried out using CINAHL, MEDLINE, PsycARTICLES, PsycINFO, Embase, and Ovid Nursing Database for studies: published up to April 2019; including people diagnosed with POAG; and assessing visual function or QoL in response to changing lighting/ luminance levels or glare. Two researchers independently screened studies for eligibility. Data were extracted from eligible studies regarding study design, participant characteristics, outcomes, and results. Quality of included studies was critically appraised. Of 8437 studies, 56 eligible studies were included. Studies investigated the effects of lighting on the following domains among people with POAG: QoL (18/56), psychophysical measures (16/56), functional vision (10/56), activities of daily living (10/56), and qualitative findings (2/56). POAG negatively affects low-luminance contrast sensitivity, glare symptoms, and dark adaptation time and extent. In vision-related QoL questionnaires, people with POAG report problems with lighting, glare, and dark adaptation more frequently than any other domain. These problems worsen with progressing visual field loss. Early-stage POAG patients experience significantly more difficulties in low-luminance or changing lighting conditions than age-matched controls (AMCs), challenging perceptions of early-stage POAG as asymptomatic. However, performance-based studies seldom show significant differences between POAG participants and AMCs on tasks simulating daily activities under non-optimal lighting conditions. Further research with larger samples is required to optimise ambient and task-oriented lighting that can support patients' adaptation to POAG.
\end{abstract}

\section{Introduction}

Glaucoma refers to a heterogeneous group of diseases which damage the optic nerve and visual field (VF). Globally, glaucoma is the most frequent cause of

Supplementary information The online version of this article (https:// doi.org/10.1038/s41433-019-0679-5) contains supplementary material, which is available to authorised users.

David P. Crabb

David.Crabb.1@city.ac.uk

1 Division of Optometry and Visual Science, School of Health Sciences, City, University of London, London, UK

2 Patient representative, London, UK

3 Department of Ophthalmology, Queen Alexandra Hospital, Portsmouth, UK irreversible blindness, with an estimated age-standardised prevalence of $\sim 3.5 \%$ among people aged 40 or older [1].

Primary open-angle glaucoma (POAG) comprises $74 \%$ of all glaucoma cases [2]. By 2020, it is expected that POAG will affect around 53 million people worldwide [3]. POAG is a chronic, progressive disease which may not have obvious symptoms until significant VF loss has occurred. It can therefore remain undetected in up to half of cases; yet people with POAG may maintain good visual outcomes with prompt diagnosis and treatment [4]. Since the condition is often asymptomatic in the early stages, it is important to understand associated functional changes. This may in turn help develop new methods to raise awareness of POAG's impacts, thus facilitating help-seeking and potential diagnosis [5].

This review will include studies that consider how lighting levels affect people with glaucoma at the mild, moderate, and advanced disease stages. This is of significant clinical interest, as there is some evidence that early-stage POAG, often considered asymptomatic, may cause issues in 
Table 1 Glossary of lightingspecific terms

\begin{tabular}{ll}
\hline Term & Definition \\
\hline Glare & A visual sensation caused by excessive brightness. \\
\multicolumn{1}{c}{ Disability glare } & Reduced vision caused by light scatter from a bright source. \\
Discomfort glare & Sensation of discomfort or annoyance caused by bright light [83] \\
Luminance & Intensity of light per unit area travelling in a certain direction [84] \\
Mesopic & Mesopic conditions exist in the range between photopic and scotopic, and mesopic vision involves \\
& both rods and cones. \\
Photopic & $\begin{array}{l}\text { Photopic conditions are well-lit (e.g. outside on a sunny day), and photopic vision involves cones, } \\
\text { facilitating colour perception. }\end{array}$ \\
Scotopic & Scotopic conditions are low light conditions (e.g. outside at night under starlight). Scotopic vision \\
& involves only rods, providing limited colour perception.
\end{tabular}

non-standard lighting conditions. For example, a recent study suggests that differences in the vision of early-stage glaucomatous patients compared with healthy controls may be magnified in non-optimal luminance conditions [6]. This systematic review therefore aims to draw together evidence on how different light levels affect visual function and vision-related quality of life (QoL) among people with glaucoma, from psychophysical studies, patient-reported outcome measures (PROMs) and performance-based tasks. Vision-related QoL can be considered as "the degree to which vision impacts an individual's ability to complete activities of daily living (ADL) and one's social, emotional and economic well-being" [7]. Table 1 provides a glossary of key lighting terminology used here.

\section{Methods}

A search of the electronic databases CINAHL Complete, MEDLINE Complete, PsycARTICLES, and PsycINFO (via EBSCOhost) and Embase and Ovid Nursing Database (via OVID) was undertaken. Keywords used related to (open angle) glaucoma, and a group of terms relating to lighting, brightness, darkness, glare, luminance, and photopic/ mesopic/scotopic conditions. For detailed search terms, see Appendix 1. Prospective and retrospective citation tracking was performed using Scopus and Google Scholar.

Studies considered eligible for inclusion were those that involved people diagnosed with glaucoma, and were written in English, French, or Spanish. Studies were required to include at least some participants with chronic POAG. If studies solely involved participants with other types of glaucoma (e.g. angle-closure glaucoma or congenital glaucoma), they were excluded. In addition, included studies had to consider the impact of different general or task lighting conditions on vision and vision-related QoL. For example, because "photopic" was a keyword, many articles were returned about the photopic negative response, a test using the electroretinogram (ERG) which can detect retinal ganglion cell dysfunction. Such articles were not included, because this is a very specific clinical use of light that is distinct from how photopic environmental conditions affect people with POAG. Studies were also excluded if their main interest related to colour, such as how specific wavelengths or coloured light (e.g. blue light) may affect people with open-angle glaucoma.

Review articles were excluded, as were studies where only an abstract was published (e.g. conference proceedings). However due to the heterogeneity of the phenomena under investigation, and the pragmatic orientation of this review, studies with only abstracts available are considered in Appendix 4, Supplementary Table 4, to avoid omitting potentially useful insights.

Two authors (JE and LJ) screened studies using Covidence systematic review software (Veritas Health Innovation Ltd, Melbourne, Australia; available at www. covidence.org) to assess eligibility. In the case of disagreements unresolved through discussion, a third author (DJT) was consulted. Relevant information (e.g. publication details, characteristics of participants, study design, outcomes measured, study results, and conclusions) from eligible papers was entered into a data extraction table.

Studies were assessed for quality using Kmet et al. Standard Quality Assessment Criteria for Evaluating Primary Research Papers from a Variety of Fields [8]. This quality appraisal tool was chosen because of the variety of quantitative and qualitative studies which are relevant to the review's questions, spanning several research fields. Full details of assessment criteria are shown in Table 2. This review is registered with the International prospective register of systematic reviews (PROSPERO; http://www.crd. york.ac.uk/prospero/; Reference CRD42018118953).

\section{Results}

Searches were run on 1 April 2019 and yielded 21,009 results (to which ten further studies were added through reference list searching). Of these, 12,582 were automatically removed as duplicates. This left 8437 studies to screen using title and abstract, of which 8125 were excluded and 312 articles were assessed for full-text eligibility. Many studies were excluded at the title and abstract screening stage because they involved animals or concerned aspects 
Table 2 Kmet et al. [8] quality assessment checklists

\begin{tabular}{|c|c|}
\hline Quantitative studies & Qualitative studies \\
\hline 1. Question/objective sufficiently described? & 1. Question/objective sufficiently described? \\
\hline 2. Study design evident and appropriate? & 2. Study design evident and appropriate? \\
\hline $\begin{array}{l}\text { 3. Method of subject/comparison group selection or source of information/input variables } \\
\text { described and appropriate? }\end{array}$ & 3. Context for the study clear? \\
\hline 4. Subject (and comparison group, if applicable) characteristics sufficiently described? & 4. Connection to a theoretical framework/wider body of knowledge? \\
\hline 5. If interventional and random allocation was possible, was it described? & 5. Sampling strategy described, relevant and justified? \\
\hline 6. If interventional and blinding of investigators was possible, was it reported? & 6. Data collection methods clearly described and systematic? \\
\hline 7. If interventional and blinding of subjects was possible, was it reported? & 7. Data analysis clearly described and systematic? \\
\hline $\begin{array}{l}\text { 8. Outcome and (if applicable) exposure measure(s) well defined and robust to measurement/ } \\
\text { misclassification bias? Means of assessment reported? }\end{array}$ & 8. Use of verification procedure(s) to establish credibility? \\
\hline 9. Sample size appropriate? & 9. Conclusions supported by the results? \\
\hline 10. Analytic methods described/justified and appropriate? & 10. Reflexivity of the account? \\
\hline \multicolumn{2}{|l|}{ 11. Some estimate of variance is reported for the main results? } \\
\hline \multicolumn{2}{|l|}{ 12. Controlled for confounding? } \\
\hline \multicolumn{2}{|l|}{ 13. Results reported in sufficient detail? } \\
\hline 14. Conclusions supported by the results? & \\
\hline
\end{tabular}

For each question, the checklist authors provide guidance on study aspects which should be considered when making a decision. For example, on Quantitative checklist Question 4 (Subject characteristics sufficiently described?), to score a Yes (2), the study in question must provide at least the age and sex of control participants

of glaucoma unrelated to lighting. Ultimately, 56 full-text studies were selected for inclusion. The study selection process is shown in a PRISMA diagram in Fig. 1. Fifty-four of the 56 studies $(96 \%)$ were quantitative, and two $(4 \%)$ were qualitative.

Quality appraisal was conducted on 55 studies. One study [9] could not be quality assessed as it dated from 1929 and was not presented in a format that allowed for comparison with other studies. The lowest score was 0.67 and the highest was 1.00 (i.e. all responses to relevant questions in the Kmet et al. criteria was Yes). Frequent issues in quantitative studies were limited information about subject/ comparison group selection and limited description of group characteristics (Fig. 2). Appendix 2, Supplementary Table 1 and Supplementary Table 2 show quality appraisal results for the 53 quantitative and 2 qualitative studies respectively.

For full details of included studies, see the data extraction table (Appendix 3, Supplementary Table 3). The subsequent overview of study findings is organised according to main outcome domain. Figure 3 shows the different domains of the studies included in this review, the most frequent of which was QoL (32\% of studies), followed by psychophysical measures (29\%). The vast majority of the included studies were case-control (48\%) and cross-sectional (41\%) studies.

\section{Quality of life (QoL)}

Eighteen studies (32\%) explored how lighting conditions affected QoL among people with glaucoma. Eleven of the eighteen $(61 \%)$ studies were cross-sectional while seven (39\%) were case-control studies. In total, 2354 participants

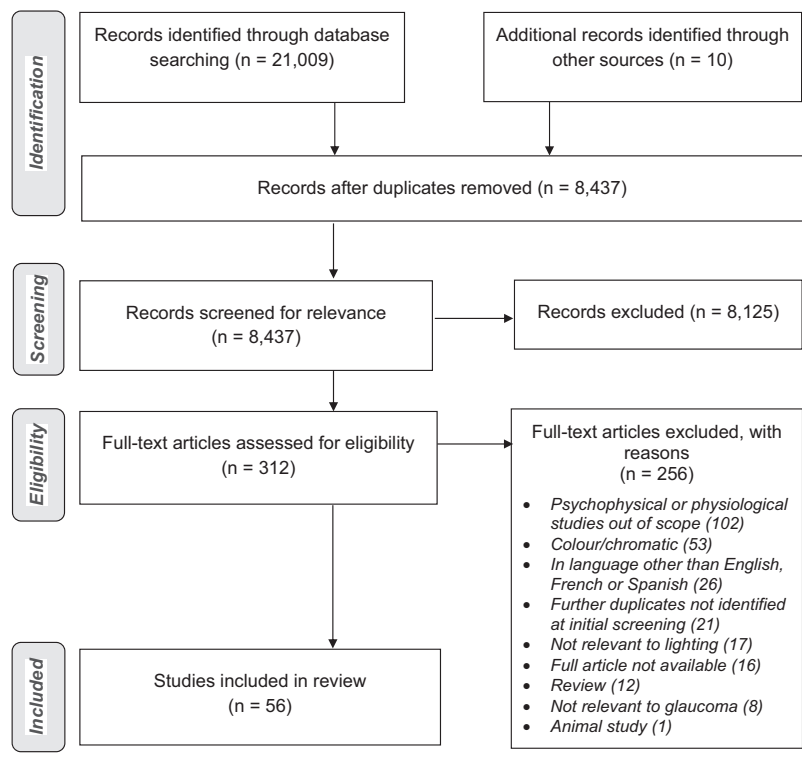

Fig. 1 PRISMA diagram showing study selection process

with glaucoma were involved in the 11 cross-sectional studies. In the case-control studies, there were 708 participants with glaucoma and 539 control participants. All the studies in this section explored QoL using PROMs, including both novel questionnaires and ones extensively developed, tested and validated for use [10].

Twelve of the 18 QoL studies explicitly excluded individuals with "visually significant", "clinically significant", or "dense" cataracts. Five cross-sectional studies provided no information about whether participants were excluded on the basis of cataracts, while in one case-control study six of the 68 glaucoma cases also had cataracts [11]. The majority of case-control studies controlled for age when analysing their 


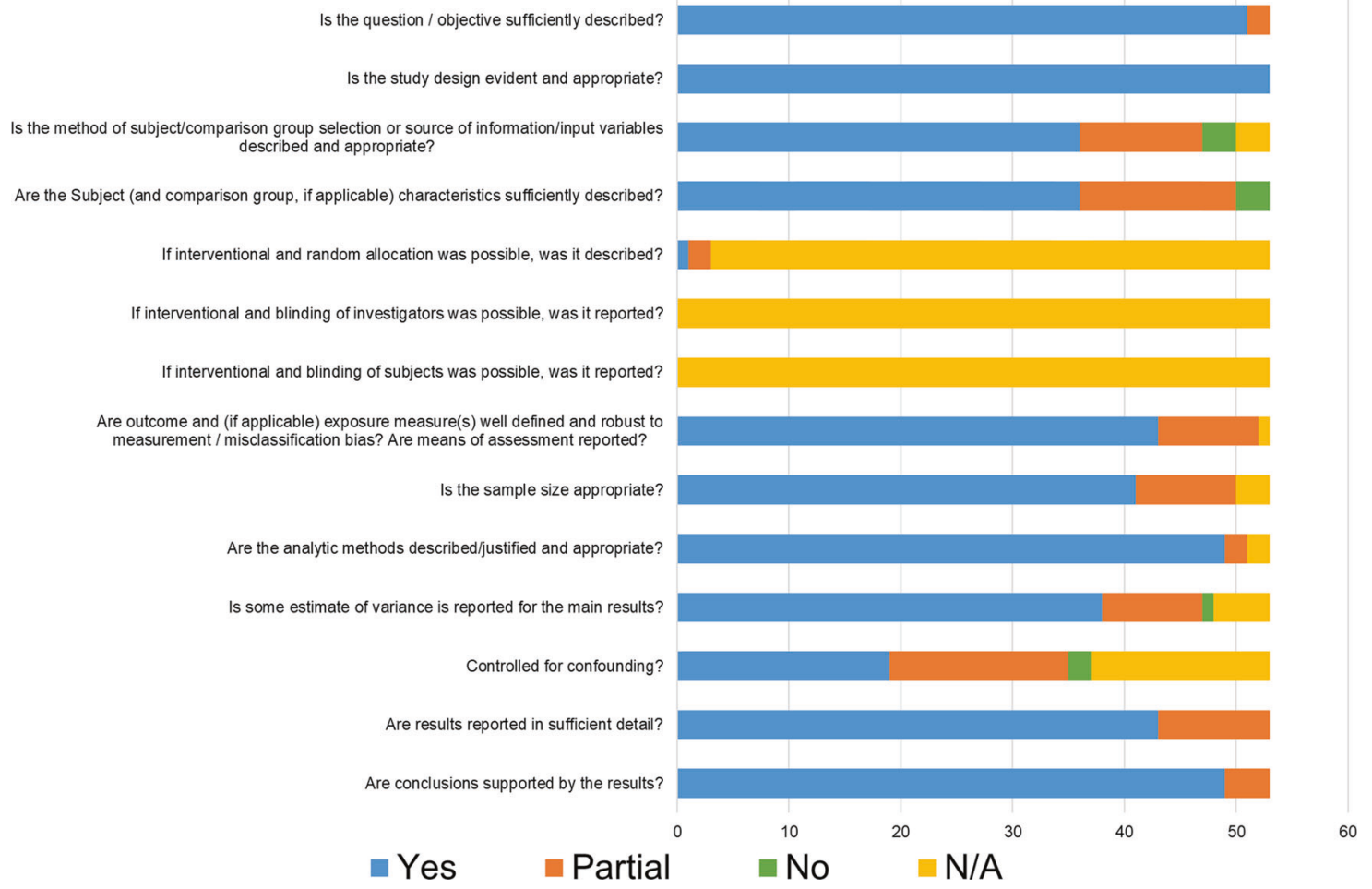

Fig. 2 Quantitative study $(N=53)$ quality appraisal results

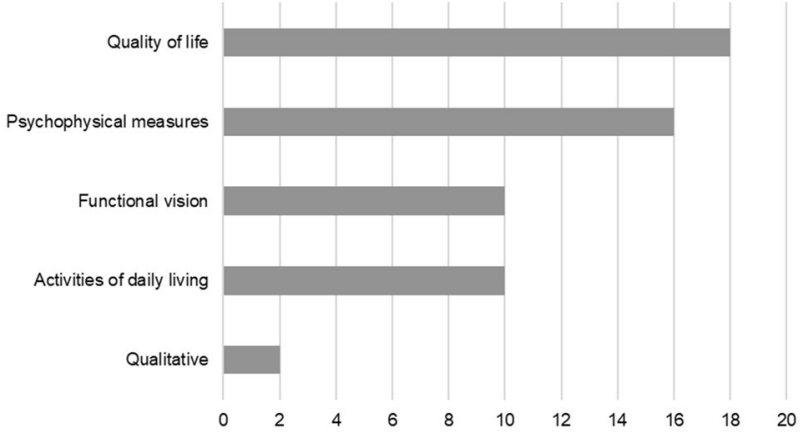

Fig. 3 Thematic domains considered in the included studies

main results, by matching groups by age and/or by adjusting for the influence of age. However, in two studies with significant differences between glaucomatous and control participants [12, 13], the review authors could not establish whether the specific lighting-relevant findings were adjusted for age.

\section{Novel questionnaires}

Nelson et al.'s study of 63 glaucoma patients found significant differences in adjusting to bright lighting $(P=0.02)$ and disability glare $(P=0.02)$ when comparing participants with mild/moderate glaucoma against those with advanced glaucoma (severe binocular VF loss) [14]. There was also a marginal, though non-significant difference in adaptation, when transitioning from a bright to dark room or vice versa $(P=0.055)$. In the authors' questionnaire on visual disability when performing daily activities, the most commonly reported problems were glare (reported as a difficulty by $70 \%$ of participants), and adaptation to different lighting levels (reported by $54 \%$ ).

The Glaucoma Symptom Scale was developed by Lee et al. using data from 147 participants with glaucoma and 44 individuals without eye disease [15]. Of the glaucomatous participants, $120(82 \%)$ reported problems seeing in the dark, vs. 14 (32\%) in the control group. Fifty-eight (39\%) glaucomatous participants reported haloes around lights, vs. six (14\%) participants in the control group. Sixty-nine (46\%) glaucomatous participants had problems seeing in daylight, vs. seven (16\%) in the control group. There was a significant difference in age between glaucomatous and control participants $(P<0.01)$, though multivariate linear regression adjusted for age still showed significant differences $(P<0.001)$ between groups on these questionnaire items. The multivariate model also controlled for presence of cataract, although there were few cataract cases and their inclusion did not substantively affect overall scores, even without correction.

A later study by Mogil et al. [16] adapted validated questionnaires such as the National Eye Institute Visual Function Questionnaire (NEI-VFQ) and Glaucoma Quality of Life (GQL-15) to create a new questionnaire with five domains: general eyesight, visual symptoms, activities, 
socioeconomic factors, and ocular symptoms. This was administered to 152 glaucomatous participants, including 97 with POAG. In the visual symptom domain, difficulty seeing in the dark was the second-most common concern (reported by $21 \%$ of participants) and glare the third most common (reported by 15\%). However, a non-lightingspecific concern, blurry vision, was the most common visual symptom (32\%). Difficulty "adjusting to changes in light settings" was reported by $4 \%$ of participants.

\section{Glaucoma quality of life (GQL-15)}

The GQL-15 questionnaire was first compiled and tested by Nelson et al. [17]. The authors found that glare disability, as tested with the brightness acuity test, correlated moderately with summary score on the GQL-15 $(r=-0.41 ; P<$ 0.001). Furthermore, dark adaptation, as tested using the Goldmann Weekers Dark Adaptometer, correlated moderately with improved scores on the GQL-15 $(r=0.34, P=$ 0.007). Out of the psychophysical tests used by the authors, only the correlation between GQL-15 summary score and Pelli-Robson contrast sensitivity was stronger $(r=-0.45$; $P<0.001)$. The authors suggest that of the four different GQL-15 subscales (central and near vision; peripheral vision; glare and dark adaptation; and outdoor mobility), the glare and dark adaptation questions could best distinguish between glaucoma severity group (mild, moderate or severe). Glare and dark adaptation showed lower (worse) scores than the other three subscales at each glaucoma severity level. There were no significant differences in age between groups, and results were adjusted for the influence of small inter-group differences in age.

The GQL-15 was used to compare a group of 121 participants with glaucoma (subdivided into mild, moderate and severe) with 31 healthy controls [12]. There was no significant difference in scores on the glare and dark adaptation scale between participants with mild glaucoma and controls, whereas the glaucoma participants were significantly more compromised than controls on all the other three GQL-15 subscales (central and near vision, peripheral vision, and outdoor mobility). Nonetheless, scores were consistently higher/worse for controls and glaucoma participants at each stage of severity on the glare and dark adaptation subscale than for any other subscale. Controls were on average 7 years younger than glaucoma participants $(P<0.001)$.

The finding that glare and dark adaptation emerges for both glaucoma participants (at each disease stage) and agematched controls as the most problematic of the four GQL-15 subscales has been found in other studies, such as Onakoya et al. [18]. In addition, when a Chinese version of the GQL-15 was administered to 508 glaucoma patients, the worst scores (out of the four subscales) were reported for glare and dark adaptation, closely followed by central and near vision [19].
In a Serbian translation of the GQL-15, the glare and dark adaptation subscale showed the worst score, with no significant difference between mild $(N=101)$ and moderate $(N=38)$ glaucoma stages, but with advanced glaucoma $(N=$ 38) participants showing significantly worse scores [20]. Another translation of the GQL-15 into Chinese found that the most problematic activities related to lighting transitions and dark adaptation. Across glaucoma severity, participants had worst scores for the questionnaire items "adjusting to bright lights" and "going from a light to a dark room or vice versa" [21]. The third worst score was for "seeing at night".

Studies by Aspinall et al. nuanced this finding that glare and dark adaptation is the most consistently problematic subscale on the GQL-15, by exploring how glaucoma patients prioritise their QoL concerns. Aspinall et al. found that lighting and glare were the most frequently reported problems, but third in a priority list after central vision and outdoor mobility [22]. An earlier study by Aspinall et al. with a different sample of patients similarly illustrated that despite their frequency, the subjective importance of glare and dark adaptation problems may be relatively low [23]. The authors suggest that the low relative impact of glare and dark adaptation on QoL may be explained by the feasibility of accommodating to the changes through behavioural or environmental modifications. The authors also found that of the different attributes of vision-related QoL, contrast sensitivity only had an effect on the prioritisation of the glare and dark adaptation subdomain $(P=0.038)$.

A QoL assessment among participants in the Collaborative Initial Glaucoma Treatment Study (CIGTS) came to a similar conclusion: that lighting and glare issues may be frequent concerns, but not necessarily the most bothersome [24]. The authors found that at baseline of enrolment into CIGTS, over $40 \%$ of participants experienced problems with bright light and light-to-dark adaptation, which were the most frequently reported problems. Furthermore, almost $30 \%$ of participants reported problems seeing in dark places. However, rating their symptoms on a 1 (not at all) to 5 (a lot) scale of how bothersome symptoms were, participants' worst symptoms related to visual distortion (mean bothersome score: 4.1) and distant vision (mean bothersome score 3.8), compared with a mean score of 3.5 for bright light, 3.2 for dark adaptation, and 3.3 for seeing in the dark.

\section{Glaucoma activity limitation (GAL-9)}

One study by Skalicky et al. included 200 participants with glaucoma, 73 of whom also had some degree of age-related macular degeneration (AMD) [13]. The authors found that adjusting to dim lights was one of the GAL-9 items which was significantly more difficult for glaucoma patients without AMD than those with AMD $(P=0.04)$. All other GAL-9 
items with reference to light ("Walking after dark"; "Seeing at night"; and "Going from light to dark room or vice versa") were all more difficult for study participants with glaucoma alone, compared with those with both glaucoma and AMD, although these differences were not statistically significant ( $P=0.20,0.42$, and 0.11 respectively).

A hospital-based study with 50 glaucoma participants showed that among GAL-9 items, "adjusting to dim lights" and "going from light to dark room and vice versa" had a relatively high difficulty score. However of all GAL-9 domains, "Adjusting to dim lights" had the weakest correlation with VF damage in the central 10 degrees $(r=-0.147, P=0.309)$ [25].

\section{Comparisons between different PROMs}

A study comparing the GQL-15 with the NEI-VFQ-25 among 132 glaucoma participants and 132 age-matched control participants found a strong correlation between the glare and dark adaptation subscale of the GQL-15 and the distance activities domain of the NEI-VFQ-25 [26]. The driving domain of the NEI-VFQ-25 most strongly correlated with the glare and dark adaptation subscale out of the four GQL-15 subscales $(r h o=-0.64, P<0.01)$. Glare and dark adaptation GQL-15 scores became higher/worse as glaucoma severity increased, particularly when comparing mild and severe. A moderate association was found between mean deviation, and glare and dark adaptation scores $(r h o=0.374)$.

Kumar et al. [27] compared two glaucoma-specific PROMs, the GQL-15 and the 10-item questionnaire developed by Viswanathan et al. [28], with the NEI-VFQ25 (a generic ophthalmology PROM). In total, 140 participants with glaucoma (49 with mild glaucoma, 55 with moderate glaucoma, and 36 with severe glaucoma) completed all three instruments. The authors found that scores from the three instruments correlated especially well in the domain of glare and dark adaptation, as well as peripheral vision. When comparing the NEI-VFQ-15 subscales and GQL-15 subdomains, the NEI-VFQ-15 driving subscale had a strong correlation with the GQL-15's glare and dark adaptation subdomain $(r=-0.62)$.

Wren et al. compared the NEI-VFQ with the Visual Activities Questionnaire (VAQ) among participants enrolled in the CIGTS [29]. Of the eight subscales on the VAQ, the light-dark adaptation subscale was the most problematic for participants, followed by glare disability. Light-dark adaptation on the VAQ correlated most strongly with Distance Activities $(r=0.56)$ and Near Activities $(r=0.53)$ on the NEI-VFQ, while glare disability correlated most with distance activities $(r=0.51)$ of all the NEI-VFQ subscales. The driving subscale of the NEI-VFQ only had moderate correlations with light-dark adaptation $(r=0.44)$ and glare disability $(r=0.43)$ on the VAQ.
Sherwood et al. used several questionnaires with 56 participants with glaucoma and 54 healthy controls (who did not differ significantly in age, $P=0.10$ ) [11]. They used the Activities of Daily Vision Scale (ADVS), and of the six subscales (day vision; night vision; far vision; near vision; glare; overall vision), the glare item showed the greatest difference in mean score between patients and controls. Investigating correlations between ADVS and Medical Outcomes Study Test (MOS-20) scores, glare had significant associations with the physical, role, mental health and general health subscales of MOS-20, although was not significantly correlated with the social and pain MOS-20 domains.

\section{Psychophysical measures}

Sixteen included studies (29\% of the total) considered how specific lighting conditions affect psychophysical outcomes such as contrast sensitivity, glare, and dark/light adaptation. These studies in total involved 517 participants with diagnosed glaucoma, and 205 participants with suspected glaucoma. The majority $(14 / 16=88 \%)$ of the psychophysics studies were case-control studies, while two were cross-sectional studies.

Studies considering how glaucoma affects psychophysical measures such as glare and dark adaptation generally considered how age and presence of cataracts could influence results. Among the 14 case-control studies, five studies reported mean ages of glaucoma and control groups which were clearly matched; five studies adjusted for age at analysis stage; while four only provided minimal information on participant age (e.g. an indicative age range). Regarding cataract, three of the 16 studies expressly excluded participants with cataracts, while two studies deliberately included participants with cataracts. Five studies more broadly excluded people with "eye abnormalities", while two studies excluded individuals who had undergone previous cataract surgery. Four studies gave no indication of having excluded individuals with cataracts.

\section{Contrast sensitivity (CS)}

Using luminance-modulated gratings, CS thresholds at all spatial and temporal frequencies have been found to be significantly poorer among people with early to moderate stage OAG relative to healthy age-matched controls [30].

Foveal CS is lower in glaucomatous eyes than eyes of agematched controls in both mesopic and photopic conditions, even in glaucomatous participants with good visual acuity [31]. For example, foveal CS, across luminance conditions, has been found to be $0.4 \log$ units lower for glaucomatous participants than for controls (age-adjusted $P<0.001$ ) [32]

However, differences in CS between healthy participants and people with glaucoma appear more pronounced in mesopic than photopic lighting conditions [33]. Even when 
differences in CS between glaucoma participants and agesimilar controls are fairly modest overall, the difference is greater in lower luminance conditions [34]. Furthermore, differences between glaucoma participants and age-matched controls in $\mathrm{CS}$ in the peripheral VF are most pronounced at low-luminance levels [32].

\section{Glare}

Glaucomatous damage has been found to be moderately positively correlated with increased glare factor $(r h o=$ $0.485 ; P=0.01$ ), with glare factor being the difference between $\log C S$ with glare and $\log C S$ without glare [35]. It should be noted, however, that this finding comes from a study in which all participants had cataract.

Disability glare among people with open-angle glaucoma has also been linked with macular pigment levels. For example, one study by Siah et al. [36] found that 54 of 88 glaucomatous participants (61\%) complained of glare symptoms. Among these participants reporting glare symptoms, lower macular pigment optical density was found at all retinal eccentricities (for $0.25^{\circ}$ and $1^{\circ}, P=0.05$ each; for $\left.0.5^{\circ}: P=0.04\right)$. There was no difference in age between participants with and without glare symptoms $(P=0.51)$; the authors controlled for the presence of mild cataract and excluded individuals with moderate-to-significant cataract.

\section{Dark adaptation}

Much of the knowledge regarding dark adaptation among people with glaucoma is based on studies from the earlyand mid-20th century.

Derby et al. showed that dark adaptation was abnormal in healthy eyes of participants who had a confirmed glaucoma diagnosis in their other eye [9]. The authors attempted to recruit controls of similar age to the glaucomatous participants (between 50 and 70) although no exact data are provided. Derby et al. also suggest that changes in the minimum amount of light which the eye can perceive could be one of the earliest signs of glaucoma.

It was thus hypothesised that the dark adaptation thresholds of glaucoma patients were reached later and were higher than in healthy eyes. Subsequently, Zuege and Drance showed that eyes with advanced glaucoma could be distinguished from age-matched healthy eyes by using the dark adaptation threshold ratio 15:30 degrees from fixation [37].

Relating to problems with dark adaptation experienced by people with POAG is the loss of scotopic sensitivity. In one study by Drum et al., participants with suspected glaucoma (no measurable VF loss, but intraocular pressure $>20 \mathrm{mmHg}$ ) and participants with confirmed glaucoma had elevated scotopic and photopic adaptation thresholds. However, among participants with confirmed glaucoma, the scotopic threshold elevation was significantly greater than the photopic threshold elevation (i.e. loss of scotopic sensitivity was greater than loss of photopic sensitivity). In addition, it was also found that the localised scotomas of glaucomatous participants were of similar depth in scotopic and photopic conditions, but in scotopic conditions, scotomas were spread out more "diffusely" across the VF [38]. When stratified by age, Drum et al. found that in their $<40$ age bracket, glaucomatous and control participants were indistinguishable, while in the 40-60 age bracket, inter-group differences were only seen for the scotopic condition.

Progressing optic nerve damage underpins decreased dark adaptation among people with glaucoma. This has been demonstrated in a study involving participants with glaucomatous and non-glaucomatous optic nerve atrophy [39]. While the authors found that the extent and velocity of dark adaptation decreases with increasing age, several measurements were significantly worse among people with glaucoma after controlling for age, and even worse with the non-glaucomatous optic nerve atrophy group (who had more severe optic nerve damage than the glaucoma group).

Bierings et al. recently showed that age-adjusted dark adaptation times are similar between glaucoma participants and controls, with only marginally (statistically non-significant) longer times among glaucoma patients relative to controls $(P=0.10$ for $5 \log$ unit luminance change, and $P=$ 0.14 for $6.5 \log$ unit luminance change) [40]. In keeping with earlier studies, they found that the dark adaptation curve for glaucoma participants has a lower CS plateau. To explain these results in line with previous findings that people with glaucoma may struggle to adjust to low-luminance conditions, the authors suggest that glaucoma participants may not strictly take longer to dark adapt to their plateau; however, because the CS plateau is lower for glaucoma participants, they will take longer when dark adapting to reach their minimum CS required for adequate everyday vision [40].

\section{Light adaptation}

In contrast to the numerous studies focused on dark adaptation, only one included study considered light adaptation, examining 23 glaucoma participants and 51 controls [40]. The study authors were unable to measure light adaptation times because of how quickly this occurred. However, in line with findings on dark adaptation, they found that after light adaptation, glaucoma participants have a lower CS plateau than healthy control participants (after adjusting for age).

\section{Visual evoked potentials (VEP)}

Arvind et al. explored the specific luminance aspects of the blue-on-yellow (BonY) multifocal visual evoked potential as a 
tool for identifying early glaucoma [41]. This study found that using a low-luminance contrast achromatic (LLA) stimulus worked as effectively as BonY in identifying early glaucoma, while a high-luminance contrast achromatic stimulus performed significantly worse than LLA and BonY in identifying VF problems. They conclude that the low-luminance contrast aspects of the BonY stimulus specifically may explain BonY's usefulness in detecting early glaucoma.

\section{Retinal function}

One study used the modified global flash multifocal ERG paradigm with luminance modulation, which allows adaptive changes in the retina to be measured [42]. The authors found that in response to a global flash stimulus at different luminance levels, participants with glaucoma had a reduced response compared with age-matched controls which correlated with glaucomatous VF damage $(r=0.58, P<0.0001)$.

In response to flash ERG after dark adaptation, glaucoma patients showed longer implicit times than age-matched controls at several flash intensities, but especially for high intensity flash [43].

A retinal sensitivity test (using flashing white light) performed under scotopic conditions has been shown to discriminate between healthy and glaucomatous participants. The study involved participants with suspected, as well as confirmed, glaucoma, and the test effectively indicated signs of early optic nerve injury among glaucoma suspects [44].

\section{Functional vision}

Ten studies (18\%) focussed on how functional vision changes under different lighting conditions. Five of the ten studies were case-control studies, three were cross-sectional and two were descriptive case series. In the case-control studies, there were 355 participants with confirmed glaucoma and 285 controls. There were 359 glaucomatous participants in the two cross-sectional studies, and 98 glaucoma participants in the two descriptive case series.

\section{Functional difficulties linked to lighting conditions}

Among participants with early or moderate glaucoma (33 of 99 study participants), the most commonly reported visual symptoms were needing more light $(58 \%)$, blurry vision (52\%), and seeing glare (52\%) [45]. When considering all participants in this study, needing more light was reported as a symptom by $57 \%$ of participants, blurry vision by $55 \%$ and seeing glare by $46 \%$.

The assessment of key markers of visual function among people with glaucoma has been shown to be dependent on lighting conditions. One study compared measures such as distance and near visual acuity (VA) and CS in the clinic and at home, with glaucomatous participants and healthy controls [46]. Overall, $29 \%$ and $22 \%$ of glaucoma participants read at least two more lines of a distance VA chart and near VA chart, respectively, in the clinic than at home. Overall, $10 \%$ of glaucoma participants read at least two triplets of a Pelli-Robson chart better in the clinic than at home. Multivariable regression analysis demonstrated that lighting levels were the strongest single factor associated with improved visual performance in the clinic. Across the whole sample of glaucoma and healthy participants, at least $85 \%$ of home lighting levels assessed were lower than recommended levels. Among glaucomatous participants, there was no statistically significant difference in lighting levels by glaucoma severity. This finding is reaffirmed by Yonge et al., who found that the individuals with more severe glaucoma symptoms were no more likely to adapt their home lighting than those with less advanced symptoms [47].

\section{Functional difficulties linked to glare}

A descriptive study in an Australian low-vision clinic reported the high prevalence of self-reported glare problems, with $34 \%$ of those whose low vision is primarily linked to glaucoma reporting being greatly affected by glare, and an additional $38 \%$ of patients being moderately affected [48]. Prevalence of glare problems was even higher among those with low vision whose secondary cause was glaucoma; with these patients, $49 \%$ were greatly affected and $29 \%$ moderately affected by glare.

\section{Functional difficulties linked to transitions in lighting or luminance}

One recent questionnaire study by Bierings et al. compared the responses of 178 glaucomatous and 182 control participants to fifteen questions about visual function when performing activities in conditions such as outdoor at night (low luminance), outdoor on a sunny day (high luminance), and sudden increases or decreases in luminance level [6]. Participants were considered to have visual complaints if they responded to such questions with either 'A lot of difficulty', 'Extreme difficulty', or 'Stopped doing this because of my eyesight'. The authors found that $4 \%$ of participants with glaucoma had visual complaints in optimal luminance vs. $0 \%$ of control participants $(P=0.02)$. Meanwhile $48 \%$ of participants with glaucoma vs. $6 \%$ of controls $(P<$ 0.001 ) experienced visual problems in low-luminance conditions. The percentages of glaucoma participants vs. controls with visual complaints was respectively 22 vs. $1 \%$ for high luminance $(P<0.001), 32$ vs. $1 \%$ for a sudden decrease in luminance $(P<0.001)$ and 25 vs. $3 \%$ for a sudden increase in luminance $(P<0.001)$. The largest differences between glaucoma participants and controls were 
found for the low-luminance questionnaire items. Overall, $48.4 \%$ of glaucoma participants reported complaints for "Seeing outside at night when there is no moonlight" (compared with $6.3 \%$ of controls); $53.6 \%$ for "Walking or cycling at night on an unlit country road" (compared with $13.7 \%$ of controls); and $49.7 \%$ for "Driving at night on an unlit country road" (compared with $12.7 \%$ of controls). The study illustrates the particular difficulties experienced by many people with glaucoma when performing daily activities in dark conditions. Further, the majority $(62 \%)$ of glaucoma patients in the study were considered to have early glaucoma in their least affected eye. As such, the findings suggest that glaucoma symptoms may be exacerbated in non-standard luminance conditions even if a patient is largely asymptomatic in optimal luminance.

In contrast, an older questionnaire study by Carta et al. [49] found no significant difference between 33 POAG participants and 20 controls (with minor refractive problems or presbyopia, and whose mean age was 9.1 years less than the POAG group) on a group of questions concerning adaptation to lighting transitions and glare $(P=0.11)$ [50]. This discrepancy may result from differences in Likert-type scales used (five points in the Bierings et al. study vs. three points in the Carta et al. study), differences in sample size, and/or the subject matter of the questions and how they were presented.

A population-based study in Japan used frequency doubling technology (FDT) perimetry and a questionnaire with 10,214 participants [51], of whom 447 exhibited VF abnormalities (VFAs, as determined by the FDT perimetry), 227 of which were attributed to glaucoma. The definitive glaucomatous subgroup were significantly more likely to report difficulty seeing in dark places, when compared with visually healthy subjects (age-adjusted odds ratio $=1.55$, $95 \% \mathrm{CI}=1.11-2.17, P=0.01)$.

\section{Motion perception}

No significant differences overall have been found between the performance of POAG and age-matched control participants in their sensitivity to motion under photopic and scotopic conditions [52]. In photopic conditions, sensitivity to moving targets in both central and peripheral vision declined with age $(P<0.01)$. In scotopic conditions, sensitivity to moving targets was lower for age-matched controls and participants with POAG than for young controls, but only for peripheral and not for central vision.

\section{Object recognition}

An experimental study has been conducted to investigate how people with glaucoma, relative to healthy controls, can recognise, or classify, common objects in different lighting levels [53]. Visual categorisation tasks were employed in two different contrast conditions (medium 50\% and high $100 \%$ ). The medium contrast setting aimed to simulate suboptimal lighting conditions, such as fog, dazzling sunlight, or dusk or dawn light. There was no difference between the performance of glaucoma patients and controls when contrast was $100 \%$. Conversely, the authors found that patients with glaucoma had $7 \%$ lower accuracy $(P=0.046)$ for the medium contrast stimuli (87\% responses correct) compared with controls (94\% responses correct). Interestingly, object categorisation in this condition was impaired despite presentation of the stimuli within the intact central area of participants' VF. This suggests that in many real-world situations where contrast is unlikely to be $100 \%$, categorising objects may be more difficult for people with glaucoma than visually healthy controls, even when considering those without significant VF loss.

Another study considered detection and categorisation of face and scene images among glaucoma patients with central VF defects compared with glaucoma patients without central VF defects in two different luminance levels [54]. Two different contrast levels (10 and $2.5 \%$ ) were used to simulate twilight conditions, lower levels than in the study discussed above. For scene images, there was no main significant main effect of the luminance contrast. However, the decrease in performance moving from the detection to categorisation task in the scene experiment was marginally greater in the $2.5 \%$ contrast condition (mean difference between tasks $\pm \mathrm{SD}=1.92 \pm 0.96$ ) compared with the $10 \%$ contrast (mean $\pm \mathrm{SD}=0.96 \pm 0.85$ ) for patients without central VF defects.

\section{Use of visual aids}

Among low-vision patients with sensitivity to light whose preferences for different light filters were assessed, over $85 \%$ of the glaucoma patients chose the two filters providing the greatest light transmission [55].

\section{Activities of daily living (ADL)}

Ten (18\%) of the fifty-six studies focussed on ADL. Of these, one was focussed on home-based modifications to lighting, four on driving, three on reading, and two on mobility. Seven of the ten ADL studies were cross-sectional, and one each was a case-control, longitudinal, and randomised control trial. These ADL studies involved a total of 1674 participants.

\section{Modifying lighting in the home}

Participants with low vision (Visual acuity $<0.3$ or $6 / 18$ ) were randomised to receive standard adaptations (control group), or specific light improvements in the kitchen, hall 
and bathroom of their home over a 6-month period (intervention group) [56]. Participants' low vision resulted from several different kinds of eye disease, with only 5 of the 46 participants diagnosed with glaucoma. There were only marginal, non-significant improvements in ease of daily activities among the intervention group, although the intervention group's improvement in QoL was significant.

\section{Driving}

Lighting conditions can play a particularly significant role in making driving more difficult for people with glaucoma, irrespective of the degree of VF loss.

Using a large dataset on activity limitation involving 293 participants with different types and severity of glaucoma, researchers focused in on responses to the driving questions, about "Driving at night"; "Driving towards oncoming headlights"; and "Driving towards the sun". In contrast, more generic aspects of driving such as "Noticing when the car in front of you is speeding up or slowing down" and "Driving during the day" were considered the least difficult [57].

In a sub-study of the CIGTS, researchers compared the concerns of drivers $(N=471)$ and non-drivers $(N=84)$ living with glaucoma [58]. Problems related to glare were the most frequently reported, with over $50 \%$ of participating drivers experiencing "some difficulty" when performing tasks affected by glare. More than $20 \%$ of the glaucomatous drivers reported "often" or "always" having difficulty seeing the road at night in the rain because of headlights. The authors suggest that glare could be a particularly useful indicator of risk for visual problems while driving, particularly since glare is one of the earliest symptoms noticeable to the patient. At 54-month follow-up, drivers with moderate-to-severe bilateral VF loss reported significantly more problems driving at night than drivers with mild bilateral VF loss.

One study explored whether glaucomatous patients ceased driving specifically because of issues with lighting, dark adaptation and glare [59]. Among 99 participants, those with moderate or severe glaucoma were more likely to have discontinued driving than those with mild glaucoma (33 vs. $8 \% ; P=0.002$ ), and to have more difficulties with dark adaptation (31 vs. $10 \% ; P=0.011$ ) and glare (27 vs. $6 \% ; P=0.012$ ). Participants with self-perceived difficulty with dark adaptation or glare were not statistically more likely to give up driving than those without. However, difficulty with dark adaptation was associated with an approximately fourfold increased likelihood of experiencing difficulty driving at night (adjusted prevalence ratio $(\mathrm{PR})=$ 3.94; $P<0.0001)$ or experiencing difficulty in poor driving conditions (adjusted PR $=4.09 ; P<0.001$ ). Self-perceived glare was not associated with difficulty driving at night, but showed a marginally significant association with difficulty in poor driving conditions ( $\mathrm{PR}=4.17 ; P=0.050$ ).

In a study of real-world driving performance among 21 drivers with bilateral moderate and advanced glaucoma (compared with 38 healthy controls), participants' driving was evaluated and scored either a pass or marginal/fail [60]. No difference was found for the glaucomatous participants who passed and failed attributable to glare $(P=0.88)$, and other psychophysical measures. The only predictor of differences between passing and failing glaucomatous drivers was performance on vision-dependent psychometric tests (especially the Trail Making Test).

\section{Reading}

Three studies were included which provided some evidence of how lighting conditions impact upon people with glaucoma's reading ability.

Two studies used the Assessment of Disability Related to Vision (ADREV), a nine-task performance-based measure which includes one task of reading in reduced illumination. Evaluation of the ADREV involving 194 participants with glaucoma showed that reading in reduced illumination was similarly difficult to a test of locating objects [61]. Further analysis of ADREV results from 192 glaucomatous participants showed that there were no statistically significant correlations between any measure of VF loss and reading in reduced illumination task performance [62]. Nonetheless, an earlier version of the measure, the Assessment of Function Related to Vision (AFREV), had found that reading in dim light had a strong correlation with CS, visual acuity of the worse eye and binocular visual acuity $(r=$ $0.68,-0.69$, and -0.67 respectively, all $P<0.01$ ) [63].

Although not formally included in the review (as it does not directly focus on lighting), research has shown that when letter contrast is reduced from 100 to $20 \%$, individuals with glaucoma read significantly more slowly than healthy controls. The relevant implication of this study is that increasing luminance in which people with glaucoma read could reduce difficulties with reading [64].

\section{Mobility}

Only two included published studies focussed on mobility. However, several of the abstracts shown in Appendix 4 explore mobility in different lighting conditions, suggesting this to be an emerging research priority.

One cross-sectional study used a mobility questionnaire and performance-test developed originally for patients with retinitis pigmentosa (RP) to assess 83 glaucoma patients [65]. Comparison of the glaucoma and RP groups' responses showed that glaucoma patients reported changes 
in lighting at night causing more mobility difficulties than the RP patients. In contrast, RP patients reported more overall difficulty walking at night.

A cross-sectional study nested within a larger US-based study (the Falls in Glaucoma Study-FIGS) considered lighting and mobility [47]. Assessing fall-related hazards in the homes of 174 people with glaucoma, ambient lighting of $<300$ lux and exposed light bulbs were the most frequently encountered fall-related hazards, in $98.9 \%$ and $96 \%$ of assessed homes, respectively. There was no relationship between VF loss and better lighting, suggesting that home lighting is often not modified even as glaucoma worsens, despite the low cost of this adaptation and its potential to reduce falls.

\section{Qualitative findings}

Two studies (4\%) used qualitative data collection methods, including interviews and focus groups, involving a total of 44 participants living with glaucoma who discussed lighting-related issues in their accounts.

In focus groups conducted in 2002, participants with glaucoma discussed problems related to lighting such as seeing halos, and shared advice with fellow group participants on how to adapt to lighting difficulties and glare; these included improving lighting in the home, and wearing hats outside to reduce the discrepancy between indoor and outdoor lighting [66].

In analysis of interviews from a more recent study, participants spoke of concerns driving at night due to the bright lights of oncoming vehicles [67]. Authors identified 'the importance of lighting' as one of their main themes impacting on patients' functioning and coping behaviours. For example, some participants spoke of needing support particularly in dark environments-particularly when navigating steps or obstacles - and when transitioning between light and dark, such as entering the cinema. Sunlight was referred to as 'blinding' by one participant. Participants suggested that artificial lights such as daylight bulbs could help. One participant found a bright light installed above her sewing machine as helpful, but felt that making the adaptation involved admitting to having a problem.

\section{Trends in publishing}

There has been an increase over time in published studies considering the impact of lighting conditions on people with glaucoma. These studies only represent a very small proportion of the total research in glaucoma. A PubMed search for articles with 'glaucoma' in the title yields 11726 results since the beginning of 2010 alone; while in that same period just 29 articles $(0.25 \%)$ were published that were relevant to lighting and included in this review.

\section{Discussion}

This review shows that lighting transitions, glare and dark adaptation clearly present problems for both the vision and QoL of people with glaucoma. They emerge as some of the most consistent and frequent symptoms among people with POAG. Studies using the GQL-15 with large numbers of participants and in different geographical contexts consistently find glare and dark adaptation to be the most commonly problematic subscale for people with glaucoma. Yet the QoL literature suggests that while difficulties relating to lighting and glare are frequently expressed, they are not perceived by patients to be the most bothersome symptoms. Indeed, Spaeth et al. suggest in a review of the glaucoma and QoL literature that a 'threshold' of dark adaption and glare disability may be reached as glaucoma progresses, beyond which the issue ceases to increase in importance [68]. Where QoL studies have explored associations between scores on the glare/dark adaptation domain and psychophysical measures, the only reliable correlation appears to be with CS [17, 23].

Evidence regarding the impact of different lighting conditions on psychophysical measures and functional vision suggests that transitioning from light to dark or vice versa is difficult for people with glaucoma. This is also borne out by qualitative studies [67]. Although a recent study from Bierings et al. suggests that people with glaucoma may not strictly take longer to dark adapt than controls [40], most evidence suggests people with glaucoma show diminished classical dark adaptation than healthy age-matched controls (AMCs) [37-39]. Bierings et al.'s questionnaire about visual performance when transitioning between different levels of ambient illumination also showed very large significant differences between glaucoma patients and AMCs [6]. Nonetheless, when glaucoma patients and AMCs undertake performancebased measures in non-optimal (e.g. scotopic) lighting on tasks such as motion perception [52], or object detection or categorisation $[53,54]$, the differences are not significant or only show small effects. A similar pattern is found in studies considering driving, with over $50 \%$ of glaucomatous drivers reporting difficulties linked to glare [58] and with problems with dark adaptation associated with a four-times higher likelihood of difficulties with night driving [59]. However when real-world driving behaviour of glaucoma patients and age-matched controls was compared, glare and other psychophysical measures did not seem to affect performance [60]. Many of the included performance-based studies have relatively small samples of glaucoma patients and controls, potentially limiting the statistical power to detect differences.

Notably, during the literature search, it emerged that many pertinent findings directly considering how lighting affects ADL exist only in conference abstracts (Appendix 4, 
Supplementary Table 4). The focus on mobility performance in many of these abstracts contrasts with the fact that only two published studies included focused on glaucoma, lighting and mobility. It is perhaps revealing that many of these abstracts, either from conferences or articles currently in proof/press, are recent and focus more directly on lighting issues than many of the included studies which only consider lighting conditions incidentally (e.g. as part of a more general questionnaire). It could therefore be inferred that improving understanding of how and why glaucoma patients may have difficulties in certain lighting conditions is becoming more of a priority concern in glaucoma research. This research is also becoming more feasible with facilities like Homelab, a simulated home environment with automated systems allowing real-world everyday task performance in different lighting levels to be monitored [69].

The included studies seldom focused specifically on adaptations and modifications that may help people with glaucoma cope with lighting-related issues. However, select parts of some studies do provide some helpful insights. Nelson et al. suggest that simple environmental modifications can help diminish glare problems and avoid rapid changes in lighting levels for people with glaucoma [17]. These include increasing brightness in dark areas (e.g. staircases), repainting dark walls in paler colours, installing or changing blinds or curtains, and considerate (re)design of window, glass and mirror areas to reduce glare. Other adaptations may include using dark lenses, and allowing longer for dark adaptation or ceasing to drive at night [23]. A study from a low-vision clinic suggests illuminated magnifiers may be useful aids, and hats with a visor and sunglasses may help against glare [48]. Arguably, it is the fact that adaptations are simple and low-intensity that may explain why such frequently encountered symptoms are low priority for many patients [23].

Nonetheless, the review suggests that lighting issues are frequently neglected in the clinical management of glaucoma. Some studies indicate people with glaucoma are living in homes with inadequate, potentially hazardous lighting, and that individuals with worse VF damage do not appear to use improved lighting [46, 47]. One study showed disparities in home and clinic lighting levels, showing that at least $85 \%$ of participants with glaucoma had inadequate home lighting, and as such had significantly worse visual acuity and CS in their home than when measured in clinic; this highlights the importance of clinicians discussing lighting with their patients as a means of minimising visual and functional difficulties in their everyday life. Advice is clearly needed that is personalised to the patient with glaucoma and their affected activities, for example ensuring that sufficient illumination is balanced against the need to minimise glare [56].
To the authors' knowledge, this is the first systematic review to focus squarely on lighting, glare and dark adaptation among people with chronic forms of glaucoma. A review in 2011 considered therapeutic uses of lighting for older adults, but with only a limited focus on the specific concerns of people with eye disease [70]. A 2011 systematic review of patient-reported outcomes in glaucoma considered studies which had used the GQL-15, but was more focused on methodological aspects of the PROMs' development and validation, rather than their thematic content [71]. Many of the present review's findings are supported by relevant sections of broader reviews on visual disability in glaucoma (see Table 3). In addition, reviews on vision, ageing and agerelated eye disease make clear that decreases in CS, in light to dark adaptation and in sensitivity to glare often occur in the healthy ageing eye without any underlying pathology. For example, glare has been found to cause significant difficulty for healthy older adults driving at night [72]. However glaucoma, and other age-related eye diseases, such as AMD and cataracts, can clearly aggravate such problems.

The number of studies exploring lighting in glaucoma is growing and Appendix 4, Supplementary Table 4 shows several abstracts, some of which will soon be published. Nonetheless, this review has shown there are as yet a limited number of published studies considering how naturalistic, real-world activities are affected by lighting conditions. The included studies using the ADREV and AFREV performance measures point to difficulties reading in low light in glaucoma, but only an aggregate measure for the task (and not scores at each level of illumination) are reported. Paucity of data on how luminance levels affect reading among people with glaucoma contrasts markedly with AMD research, where the effects of differing luminance levels on reading performance has been explored in both cross-sectional [73] and case-control [74] studies. An additional research gap concerns how glaucoma patients' visual acuity may change according to background luminance levels; in this review, only Bhorade et al. [46] specifically explored glaucoma participants' visual acuity in different lighting conditions. Furthermore, while there were descriptive findings regarding adaptation strategies, no research was identified evaluating how low-vision aids, assistive technologies or rehabilitation initiatives may support people with glaucoma to cope with lighting and glare difficulties.

Although no studies were formally excluded on the basis of insufficient quality, some common study limitations were identified. Only a minority of studies reported the treatments of the glaucomatous participants involved. When treatment status was reported, many patients were being treated with drops: medications that are associated with ocular surface changes and which may be associated with 
Table 3 Select findings relevant to lighting from key reviews

\begin{tabular}{|c|c|c|c|}
\hline Author(s) & Year & Title & Select findings related to lighting \\
\hline Derby et al. & 1926 & $\begin{array}{l}\text { Further studies on the light sense in early } \\
\text { glaucoma [85] }\end{array}$ & $\begin{array}{l}\text { "It is our distinct impression that an examination of the light } \\
\text { minimum... is of real value to us in making the diagnosis of early } \\
\text { glaucoma". }\end{array}$ \\
\hline Marlow & 1947 & $\begin{array}{l}\text { The field of vision in chronic glaucoma (a } \\
\text { comparison of full with reduced } \\
\text { illumination) [86] }\end{array}$ & $\begin{array}{l}\text { "The evidence presented here permits the conclusion that reduction } \\
\text { of illumination is more than sometimes useful and that it is of } \\
\text { definite value in the discovery of incipient changes as well as in the } \\
\text { amplification of known or suspected defects". }\end{array}$ \\
\hline Ramulu & 2009 & $\begin{array}{l}\text { Glaucoma and disability: which tasks are } \\
\text { affected, and at what stage of disease? [87] }\end{array}$ & $\begin{array}{l}\text { "Difficulties related to lighting such as glare and difficulty adapting } \\
\text { to different levels of light consistently ranked as the most frequent } \\
\text { complaint... The lighting conditions under which tasks are } \\
\text { performed may be even more important than the task itself". }\end{array}$ \\
\hline Medeiros et al. & 2012 & $\begin{array}{l}\text { Driving Simulation as a Performance- } \\
\text { based Test of Visual Impairment in } \\
\text { Glaucoma [88] }\end{array}$ & $\begin{array}{l}\text { "Increasingly challenging visual tasks on the [driving] simulator, } \\
\text { under low-contrast, low-luminance conditions and performed under } \\
\text { the pressure of time, could potentially reveal functional } \\
\text { impairments that would not be detected by standard visual field } \\
\text { assessment. This hypothesis remains to be investigated." }\end{array}$ \\
\hline Nassiri et al. & 2013 & $\begin{array}{l}\text { National Eye Institute Visual Function } \\
\text { Questionnaire: Usefulness in Glaucoma } \\
\text { [89] }\end{array}$ & $\begin{array}{l}\text { "Contrast sensitivity, glare sensitivity, and dark adaptation are } \\
\text { potential items that could be added to the [NEI-VFQ] questionnaire } \\
\text { to make it more responsive to changes in vision-related QoL in } \\
\text { patients with glaucoma". }\end{array}$ \\
\hline Wang et al. & 2017 & $\begin{array}{l}\text { The impact of mild, moderate, and severe } \\
\text { visual field loss in glaucoma on patients' } \\
\text { quality of life measured via the Glaucoma } \\
\text { Quality of Life-15 Questionnaire: a meta- } \\
\text { analysis [90] }\end{array}$ & $\begin{array}{l}\text { "Glare and dark adaptation did not differ significantly between } \\
\text { patients with mild and moderate visual field loss... glare and dark } \\
\text { adaptation differed significantly between patients with moderate } \\
\text { and severe glaucoma". }\end{array}$ \\
\hline Owsley et al. & 2018 & Vision and aging [91] & $\begin{array}{l}\text { "Contrast sensitivity loss tends to be more severe in older adults } \\
\text { who have one or more of the common eye chronic conditions of } \\
\text { aging mentioned earlier. In glaucoma these losses are largely } \\
\text { attributable to the loss of ganglion cells... } \\
\text { There is also evidence suggesting that glaucoma impairs rod- } \\
\text { mediated dark adaptation". }\end{array}$ \\
\hline
\end{tabular}

glare symptoms and generally reduced visual function [75]. Treatment with topical beta antagonists and glaucoma surgery are also associated with increased cataract [76, 77]. This may therefore limit the extent to which lighting issues caused by glaucoma may be clearly distinguished from treatment side effects and co-pathology. Studies employing the GQL-15 clearly suggest dark adaptation and glare are the most frequently encountered issue, even in early-stage glaucoma; this may not meaningfully reflect the clinical experience, where glaucoma is commonly seen as asymptomatic. This implies that there may be discrepancy between patients' self-reports when they consider individual isolated symptoms, and their overall visual performance where lighting may have a relatively insignificant impact. Finally, there is heterogeneity in the definition of key concepts across studies. For example, the included psychophysics studies consider dark adaptation as a process taking many minutes, while the GQL-15 domain for dark adaptation and glare is based on items such as "going from a light to a dark room", a near instantaneous process. Hence, it could be argued that 'classical' dark adaptation studies provide only partial insight into the underlying mechanism of glaucoma patients' reports of disorientation when moving from outside into a dark indoor space (e.g. a cinema). Similarly, in many of the included studies, distinctions between discomfort glare and disability glare are seldom clarified or specified. Furthermore, it is possible that the term 'glare' may be used to describe difficulty with a rapid increase in light level, or, to describe a dramatic loss in visual performance in the presence of backlight. The research landscape in lighting and glaucoma therefore appears relatively incoherent, and shows a gap in studies that integrate an understanding of the physiological and psychophysical processes with real-world clinical effects.

There are further interesting observations about the scope of the review and included articles that could be interpreted as limitations. First, as the distribution of rods is predominant mainly outside the macular area, the studies synthesised could be affected by consideration of the location of the VF damage (central or peripheral). This kind of information was rarely stated or recorded in the studies we considered; many studies provided mean deviation values as an overall measure of VF loss, but only in a small number of cases (e.g. Wolffsohn and Cochrane) was the location of participants' VF loss 
thoroughly described [48]. The parallel here is the lack of attention that is often given to the actual location and spatial extent of VF loss when assessing impact on function in any lighting condition [5]. Second, the present review has not specifically considered the mechanism of early functional deficits of glare and adaptation in glaucoma patients. For example, it has been suggested that large $(\mathrm{M})$ retinal ganglion cells are selectively damaged by glaucomatous optic nerve atrophy, and these cells are known to be particularly sensitive to luminance contrast under mesopic and scotopic conditions $[37,38,78]$. Indeed, for several decades it had been proposed that glaucomatous loss was sensitive to stimuli that preferentially stimulate the magnocellular (M) ganglion cells relative to parvocellular $(\mathrm{P})$ ganglion cells, based on the notion that $\mathrm{M}$ cells are selectively damaged in glaucoma [79]. More recent evidence has contradicted this notion; [80] for example, McKendrick et al. have identified foveal and midperipheral dysfunction of both $\mathrm{M}$ and $\mathrm{P}$ pathways among people with glaucoma [81]. Third, it is important to note that the focus of this review is investigating the effect of different lighting conditions on real-world function and vision-related QoL. Thus it should be noted that a more detailed review of psychophysical measures, such as ERG (electroretinogram) studies, is not covered by our review. Fourth, none of the included studies explored in detail how VF measurements may be affected by lighting conditions. Conventional VF testing uses bright stimuli on a dark background, which may be advantageous to detect glaucoma; however the results may be different when patients are in a bright place in their daily lives. Indeed, the outcome of VF measurement may be different when dark stimuli, instead of bright stimuli, are used [82].

This review's methodology also has a number of limitations. First, for the purposes of data extraction and quality assessment, only studies published in peer-reviewed journals were formally included in results. This is a shortcoming not only because of publication bias, but also because many of the most relevant findings exist in a grey literature form, for example as conference abstracts (Appendix 4, Supplementary Table 4). Second, the range of languages spoken in the review team only allowed for screening and inclusion of studies in English, French or Spanish (and only English articles were ultimately included). This meant that a fairly large number of seemingly relevant studies - in German (13 studies), Japanese (5), and Russian (4) in particular-were therefore excluded. Third, while included studies were required to include at least some POAG patients, the glaucoma group in some studies involved participants with other forms of glaucoma (e.g. normal-tension glaucoma, or secondary open-angle glaucoma, such as pseudoexfoliative or pigmentary glaucoma). In these studies, results were rarely disaggregated by exact glaucoma type, thereby limiting how highly specific the review's findings are to POAG. A fourth limitation relates to using the assessment criteria we chose (Kmet et al. [8]) for a heterogeneous research field. Most studies were appraised as being of high methodological quality, with a mean of 0.91 (out of 1) for quantitative studies and 0.83 for qualitative studies. However, some studies scored 1 because they elegantly replicated an existing questionnaire, such as the GQL-15 in a different population; while another more novel, complex study could score relatively low if it did not meet the precise assessment criteria. In addition, the inclusion of a study from 1929 [9] presented a significant challenge for using a tool that assesses quality according to modern scientific conventions, with the result that that study was omitted for quality assessment.

In spite of these limitations, in conclusion this review provides clear evidence that lighting conditions may cause frequent, although not always hugely problematic, difficulties for the everyday visual function, QoL and daily activities of people with glaucoma. The weight of the evidence suggests these difficulties with lighting transitions and glare exceed those experienced by healthy older adults more generally, and that the problems become worse as glaucoma severity increases. Nonetheless, tests of dark adaptation or questionnaires do not themselves allow for clear discrimination between people with glaucoma and visually healthy controls. Using naturalistic performance-based measures to assess activities of daily living under different lighting levels is becoming more feasible as a valuable area for further research. Similarly, research focussed on low-vision aids and rehabilitation also emerges as a clear priority for future research, given both the lack of studies identified on this theme and the clear evidence that the majority of glaucoma patients' home lighting is inadequate. Clinicians should be aware of the impacts of illumination on glaucoma patients and how visual function as measured in the clinic may not reflect their real-world visual performance, especially at night or under scotopic conditions.

\section{Summary}

\section{What was known before}

- Glaucoma is a progressive optic neuropathy which may have few symptoms in its early stages.

- Some evidence points to difficulties people with glaucoma may experience in extremes of lighting (e.g. very bright or very dark conditions) or when transitioning between different luminance levels (e.g. light or dark adaptation).

- Dark adaptation and glare are common concerns in patient-reported outcome measures of vision-related quality of life in glaucoma. 


\section{What this study adds}

- This systematic review, including 56 studies, is the first to focus on the impacts of lighting and luminance conditions on people with glaucoma.

- Issues with lighting, especially lighting extremes or transitions, are highly prevalent in glaucoma and become worse with increasing visual field loss. However lighting concerns do not feature among glaucoma patients' most bothersome concerns.

- Psychophysical studies suggest glaucoma negatively affects low-luminance contrast sensitivity, glare symptoms, and dark adaptation time and extent. However, performance-based studies seldom show significant differences between individuals with glaucoma and age-matched controls on tasks simulating daily activities under non-optimal lighting conditions.

Acknowledgements This review was supported by funds from an unrestricted investigator initiated grant awarded to David P Crabb by Santen. Santen had no role in the design and conduct of the study; collection, management, analysis, and interpretation of the data; preparation, review, or approval of the manuscript; and decision to submit the manuscript for publication.

\section{Compliance with ethical standards}

Conflict of interest DPC has received unrestricted research funding from Roche, Santen and Allergan. DPC has received speaker's fees from Roche, Allergan, THEA and Bayer. DPC acts as a consultant for Centervue. The remaining authors declare that they have no conflict of interest.

Publisher's note Springer Nature remains neutral with regard to jurisdictional claims in published maps and institutional affiliations.

\section{References}

1. Jonas JB, Aung T, Bourne RR, Bron AM, Ritch R, Panda-Jonas S. Glaucoma. Lancet. 2017;390:2183-93. https://doi.org/10.1016/ S0140-6736(17)31469-1

2. Quigley HA, Broman AT. The number of people with glaucoma worldwide in 2010 and 2020. Br J Ophthalmol. 2006;90:262 LP-267. http://bjo.bmj.com/content/90/3/262.abstract.

3. Tham Y-C, Li X, Wong TY, Quigley HA, Aung T, Cheng C-Y. Global prevalence of glaucoma and projections of glaucoma burden through 2040: a systematic review and meta-analysis. Ophthalmol. 2014;121:2081-90. https://doi.org/10.1016/j.ophtha. 2014.05.013.

4. Goh YW, Ang GS, Azuara-Blanco A. Lifetime visual prognosis of patients with glaucoma. Clin Exp Ophthalmol. 2011;39:766-70. https://doi.org/10.1111/j.1442-9071.2011.02559.x.

5. Crabb DP. A view on glaucoma-are we seeing it clearly? Eye. 2016;30:304-13. https://www.ncbi.nlm.nih.gov/pubmed/ 26611846.

6. Bierings RAJM, van Sonderen FLP, Jansonius NM. Visual complaints of patients with glaucoma and controls under optimal and extreme luminance conditions. Acta Ophthalmol. 2018;96:288-94. https://doi.org/10.1111/aos.13695.

7. Angeles-Han ST, Griffin KW, Harrison MJ, Lehman TJA, Leong T, Robb RR, et al. Development of a vision-related quality of life instrument for children ages 8-18 years for use in juvenile idiopathic arthritis-associated uveitis. Arthritis Care Res. 2011;63:1254-61. https://www.ncbi.nlm.nih.gov/pubmed/21678564.

8. Kmet LM, Lee RC, Cook LS. HTA Initiative \#13. Standard quality assessment criteria for evaluating primary research papers from a variety of fields. HTA Initiative. 2004. https://www.ihe. $\mathrm{ca} / \mathrm{advanced}$-search/standard-quality-assessment-criteria-for-eva luating-primary-research-papers-from-a-variety-of-fields.

9. Derby GS, Chandler PA, O'Brien ME. The light sense in early glaucoma: the smallest difference in brightness perceptible to the light adapted eye (light difference). JAMA Ophthalmol. 1929;1:692-703. 10.1001/archopht.1929.00810010718004

10. Black N. Patient reported outcome measures could help transform healthcare. BMJ Br Med J. 2013;346:f167 http://www.bmj.com/ content/346/bmj.f167.abstract.

11. Sherwood MB, Garcia-Siekavizza A, Meltzer MI, Hebert A, Burns AF, McGorray S. Glaucoma's impact on quality of life and its relation to clinical indicators: A pilot study11The authors have no proprietary interest in the development or marketing of this or a competing instrument. Ophthalmology. 1998;105:561-6.

12. Goldberop I, Clement CI, Chiang TH, Walt JG, Lee LJ, Graham S, et al. Assessing quality of life in patients with glaucoma using the glaucoma quality of life-15 (GQL-15) questionnaire. J Glaucoma. 2009;18. https://journals.lww.com/glaucomajournal/Fulltext/2009/ 01000/Assessing_Quality_of_Life_in_Patients_With.2.aspx.

13. Skalicky SE, Fenwick E, Martin KR, Crowston J, Goldberg I, McCluskey P. Impact of age-related macular degeneration in patients with glaucoma: understanding the patients' perspective. Clin Exp Ophthalmol. 2016;44:377-87.

14. Nelson P, Aspinall P, O'Brien C. Patients' perception of visual impairment in glaucoma: A pilot study. $\mathrm{Br} \mathrm{J}$ Ophthalmol. 1999;83:546-52.

15. Lee BL, Gutierrez P, Gordon M, Wilson MR, Cioffi GA, Ritch R, et al. The glaucoma symptom scale: a brief index of glaucomaspecific symptoms. JAMA Ophthalmol. 1998;116:861-6. https:// doi.org/10.1001/archopht.116.7.861. Available from

16. Mogil RS, Tirsi A, Lee JM, Tello C, Park SC. Glaucoma patient-reported concerns and associated factors. Am J Ophthalmol. 2017;178:9-17. https://doi.org/10.1016/j.ajo.2017.03.009.

17. Nelson P, Aspinall P, Papasouliotis O, Worton B, O'Brien C. Quality of life in glaucoma and its relationship with visual function. J Glaucoma. 2003;12:139-50.

18. Onakoya AO, Mbadugha CA, Aribaba OT, Ibidapo OO. Quality of life of primary open angle glaucoma patients in Lagos, Nigeria: clinical and sociodemographic correlates. J Glaucoma. 2012;21:287-95.

19. Zhou C, Qian S, Wu P, Qiu C. Quality of life of glaucoma patients in China: Sociodemographic, clinical, and psychological correlates - a cross-sectional study. Qual Life Res. 2014;23:999-1008.

20. Sencanic I, Gazibara T, Dotlic J, Stamenkovic M, Jaksic V, Bozic $\mathrm{M}$, et al. Validation of the glaucoma quality of life- 15 questionnaire in serbian language. Int $\mathrm{J}$ Ophthalmol. 2018;11:1674-84. https://www.ncbi.nlm.nih.gov/pubmed/30364180.

21. Lee JWY, Chan CWS, Chan JCH, Li Q, Lai JSM. The association between clinical parameters and glaucoma-specific quality of life in Chinese primary open-angle glaucoma patients. Hong Kong Med J. 2014;20:274-8.

22. Aspinall PA, Johnson ZK, Azuara-Blanco A, Montarzino A, Brice R, Vickers A. Evaluation of quality of life and priorities of patients with glaucoma. Investig Ophthalmol Vis Sci. 2008;49:1907-15. 
23. Aspinall PA, Hill AR, Nelson P, O'brien C, O'connell E, McCloughan L, et al. Quality of life in patients with glaucoma: a conjoint analysis approach. Vis Impair Res. 2005;7:13-26.

24. Janz NK, Wren PA, Lichter PR, Musch DC, Gillespie BW, Guire KE. Quality of life in newly diagnosed glaucoma patients. Ophthalmology. 2001;108:887-97.

25. Daruka R, Kuzhuppilly NIR, Dev S, Patil SN, Rajendraprasad S. Correlation of central field index (10-2 visual field analysis) and activity limitation with increasing severity of glaucoma using glaucoma activity limitation-9 questionnaire. Indian J Ophthalmol [Internet]. 2018;66:1098-103. https://www.ncbi.nlm.nih.gov/ pubmed/30038150.

26. Mbadugha CA, Onakoya AO, Aribaba OT, Akinsola FB. A comparison of the NEIVFQ25 and GQL-15 questionnaires in Nigerian glaucoma patients. Clin Ophthalmol. 2012;6:1411-9.

27. Kumar S, Thakur S, Ichhpujani P. The impact of primary openangle glaucoma: comparison of vision-specific (National Eye Institute Visual Function Questionnaire-25) and disease-specific (Glaucoma Quality of Life-15 and Viswanathan 10) patientreported outcome (PRO) instruments. Indian J Ophthalmol. 2019;67:83-8. https://www.ncbi.nlm.nih.gov/pubmed/30574899.

28. Viswanathan AC, McNaught AI, Poinoosawmy D, Fontana L, Crabb DP, Fitzke FW, et al. Severity and Stability of glaucoma: patient perception compared with objective measurement. JAMA Ophthalmol. 1999;117:450-4. https://doi.org/10.1001/archopht. 117.4.450.

29. Wren PA, Guire KE, Niziol LM, Janz NK, Gillespie BW, Musch DC. Contrasting the use of 2 vision-specific quality of life questionnaires in subjects with open-angle glaucoma. J Glaucoma. 2009;18:403-11.

30. Ansari EA, Morgan JE, Snowden RJ. Psychophysical characterisation of early functional loss in glaucoma and ocular hypertension. Br J Ophthalmol. 2002;86:1131-5.

31. Lahav K, Levkovitch-Verbin H, Belkin M, Glovinsky Y, Polat U. Reduced mesopic and photopic foveal contrast sensitivity in glaucoma. Arch Ophthalmol. 2011;129:16-22.

32. Bierings RAJM, de Boer MH, Jansonius NM. Visual performance as a function of luminance in glaucoma: the De Vries-Rose, Weber's, and Ferry-Porter's LawDe Vries-Rose, Weber's, and Ferry-Porter's law in glaucoma. Invest Ophthalmol Vis Sci. 2018;59:3416-23. https://doi.org/10.1167/iovs.17-22497.

33. Hertenstein H, Bach M, Gross NJ, Beisse F. Marked dissociation of photopic and mesopic contrast sensitivity even in normal observers. Graefe's Arch Clin Exp Ophthalmol. 2016;254:373-84.

34. Bierings RAJM, Overkempe T, van Berkel CM, Kuiper M, Jansonius NM. Spatial contrast sensitivity from star- to sunlight in healthy subjects and patients with glaucoma. Vis Res. 2019;158:31-9. https://doi.org/10.1016/j.visres.2019.01.011.

35. Klein J, Pierscionek BK, Lauritzen J, Derntl K, Grzybowski A, Zlatkova MB. The effect of cataract on early stage glaucoma detection using spatial and temporal contrast sensitivity tests. PLoS One. 2015;10:1-17.

36. Siah WF, O'Brien C, Loughman JJ. Macular pigment is associated with glare-affected visual function and central visual field loss in glaucoma. Br J Ophthalmol. 2018;102:929-35.

37. Zuege P, Drance SM. Studies of dark adaptation of discrete paracentral retinal areas in glaucomatous subjects. Am J Ophthalmol. 1967;64:56-63.

38. Drum B, Armaly MF, Huppert W. Scotopic sensitivity loss in glaucoma. JAMA Ophthalmol. 1986;104:712-7. https://doi.org/ 10.1001/archopht.1986.01050170102031.

39. Jonas JB, Zäch F-M, Naumann GOH. Dark adaptation in glaucomatous and nonglaucomatous optic nerve atrophy. Graefe's Arch Clin Exp Ophthalmol. 1990;228:321-5. https://doi.org/10. 1007/BF00920055.
40. Bierings RAJM, Kuiper M, Van Berkel CM, Overkempe T, Jansonius NM. Foveal light and dark adaptation in patients with glaucoma and healthy subjects: a case-control study. PLoS One. 2018;13:1-11.

41. Arvind H, Klistorner A, Grigg J, Graham SL. Low-luminance contrast stimulation is optimal for early detection of glaucoma using multifocal visual evoked potentials. Investig Ophthalmol Vis Sci. 2011;52:3744-50.

42. Chu PHW, Chan HHL, Brown B. Glaucoma detection is facilitated by luminance modulation of the global flash multifocal electroretinogram. Investig Ophthalmol Vis Sci. 2006;47:929-37.

43. Velten IM, Horn FK, Korth M, Velten K. The b-wave of the dark adapted flash electroretinogram in patients with advanced asymmetrical glaucoma and normal subjects. Br J Ophthalmol. 2001;85:403 LP-409. http://bjo.bmj.com/content/85/4/403.abstract.

44. Glovinsky Y, Quigley HA, Drum B, Bissett RA, Jampel HD. A whole-field scotopic retinal sensitivity test for the detection of early glaucoma damage. JAMA Ophthalmol. 1992;110:486-90. https://doi.org/10.1001/archopht.1992.01080160064031.

45. Hu CX, Zangalli C, Hsieh M, Gupta L, Williams AL, Richman J, et al. What do patients with glaucoma see? Visual symptoms reported by patients with glaucoma. Am J Med Sci. 2014;348:403-9. https:// doi.org/10.1097/MAJ.0000000000000319.

46. Bhorade AM, Perlmutter MS, Wilson B, Kambarian J, Chang S, Pekmezci M, et al. Differences in vision between clinic and home and the effect of lighting in older adults with and without glaucomadifferences in vision between clinic and homedifferences in vision between clinic and home. JAMA Ophthalmol. 2013;131:1554-62. https://doi.org/10.1001/jamaophthalmol.2013.4995.

47. Yonge AV, Swenor BK, Miller R, Goldhammer V, West SK, Friedman DS, et al. Quantifying fall-related hazards in the homes of persons with glaucoma. Ophthalmol. 2017;124:562-71. https:// doi.org/10.1016/j.ophtha.2016.11.032.

48. Wolffsohn JS, Cochrane AL. Low vision perspectives on glaucoma. Clin Exp Optom. 1998;81:280-9.

49. Carta A, Braccio L, Belpoliti M, Soliani L, Sartore F, Gandolfi SA, Maraini G. Self-assessment of the quality of vision: association of questionnaire score with objective clinical tests. Curr Eye Res. 1998;17:506-12.

50. Carta A, Braccio L, Belpoliti M, Soliani L, Sartore F, Gandolfi SA, et al. Self-assessment of the quality of vision: association of questionnaire score with objective clinical tests. Curr Eye Res. 2003;17:506-12.

51. Tatemichi M, Nakano T, Hayashi T, Tanaka K, Hiro H, Miyamoto $\mathrm{T}$, et al. Symptoms related to glaucomatous visual field abnormalities among male Japanese workers in a population-based setting. Acta Ophthalmol. 2012;90:546-51. https://doi.org/10.1111/j. 1755-3768.2010.01988.x.

52. Willis A, Anderson SJ. Effects of Glaucoma and Aging on Photopic and Scotopic Motion Perception. Invest Ophthalmol. 2000;41:325-35.

53. Lenoble Q, Lek JJ, Mckendrick AM. Visual object categorisation in people with glaucoma. Br J Ophthalmol. 2016;100:1585-90.

54. Roux-Sibilon A, Rutgé F, Aptel F, Attye A, Guyader N, Boucart $\mathrm{M}$, et al. Scene and human face recognition in the central vision of patients with glaucoma. PLoS ONE. 2018;13:1-19.

55. Hoeft WW, Hughes MK. A comparative study of low-vision patients: their ocular disease and preference for one specific series of light transmission filters. Optom Vis Sci. 1981;58. https:// journals.lww.com/optvissci/Fulltext/1981/10000/A_Compara tive_Study_of_Low_Vision_Patients_Their.9.aspx.

56. Brunnström G, Sörensen S, Alsterstad K, Sjöstrand J. Quality of light and quality of life - the effect of lighting adaptation among people with low vision. Ophthalmic Physiol Opt. 2004;24:274-80. https://doi.org/10.1111/j.1475-1313.2004.00192.x. 
57. Khadka J, Fenwick EK, Lamoureux EL, Pesudovs K. Item banking enables stand-alone measurement of driving ability. Optom Vis Sci. 2016;93:1502-12. https://doi.org/10.1097/OPX. 0000000000000958.

58. Janz NK, Musch DC, Gillespie BW, Wren PA, Niziol LM. Evaluating clinical change and visual function concerns in drivers and nondrivers with glaucoma. Investig Ophthalmol Vis Sci. 2009;50:1718-25.

59. Tam ALC, Trope GE, Buys YM, Yang Y, Shen C, Jin YP. Selfperceived Impact of glaucomatous visual field loss and visual disabilities on driving difficulty and cessation. J Glaucoma. 2018;27:981-6.

60. Bhorade AM, Yom VH, Barco P, Wilson B, Gordon M, Carr D. On-road driving performance of patients with bilateral moderate and advanced glaucoma. Am J Ophthalmol. 2016;166:43-51. https://doi.org/10.1016/j.ajo.2016.02.031.

61. Lorenzana L, Lankaranian D, Dugar J, Mayer J, Palejwala N, Kulkarni K, et al. A new method of assessing ability to perform activities of daily living: design, methods and baseline data. Ophthalmic Epidemiol. 2009;16:107-14. https://doi.org/10.1080/ 09286580902738142.

62. Kulkarni KM, Mayer JR, Lorenzana LL, Myers JS, Spaeth GL. Visual field staging systems in glaucoma and the activities of daily living. Am J Ophthalmol. 2012;154:445-.e3. https://doi.org/10. 1016/j.ajo.2012.03.030.

63. Altangerel U, Spaeth GL, Steinmann WC. Assessment of function related to vision (AFREV). Ophthalmic Epidemiol. 2006;13:67-80. https://doi.org/10.1080/09286580500428500.

64. Burton R, Crabb DP, Smith ND, Glen FC, Garway-Heath DF. Glaucoma and reading: exploring the effects of contrast lowering of text. Optom Vis Sci. 2012;89. https://journals.lww.com/optvissci/ Fulltext/2012/09000/Glaucoma_and_Reading__Exploring_the_ Effects_of.10.aspx.

65. Turano KA, Massof RW, Quigley HA. A self-assessment instrument designed for measuring independent mobility in rp patients: generalizability to glaucoma patients. Invest Ophthalmol Vis Sci. 2002;43:2874-81.

66. Green J, Siddall H, Murdoch I. Learning to live with glaucoma: a qualitative study of diagnosis and the impact of sight loss. Soc Sci Med. 2002;55:257-67.

67. Glen FC, Crabb DP. Living with glaucoma: a qualitative study of functional implications and patients' coping behaviours. BMC Ophthalmol. 2015;15:1-15. https://doi.org/10.1186/s12886-015-0119-7.

68. Spaeth G, Walt J, Keener J. Evaluation of quality of life for patients with glaucoma. Am J Ophthalmol. 2006;141:3-14. http://www. sciencedirect.com/science/article/pii/S0002939405008779.

69. Zenouda A, Lombardi M, Gutman E, Brasnu E, Hamard P, Sahel JA, et al. Effect of different lightning conditions on daily living activities of glaucoma patients. Acta Ophthalmol. 2016 Oct;94. https://doi.org/10.1111/j.1755-3768.2016.0554.

70. Shikder S, Mourshed M, Price A. Therapeutic lighting design for the elderly: a review. Perspect Public Health. 2011;132:282-91. https://doi.org/10.1177/1757913911422288.

71. Vandenbroeck S, De Geest S, Zeyen T, Stalmans I, Dobbels F. Patient-reported outcomes (PRO's) in glaucoma: a systematic review. Eye]. 2011;25:555-77. https://www.ncbi.nlm.nih.gov/ pubmed/21423144.

72. Kimlin JA, Black AA, Wood JM. Nighttime driving in older adults: effects of glare and association with mesopic visual function. Invest Ophthalmol Vis Sci. 2017;58:2796-803. https:// doi.org/10.1167/iovs.16-21219.

73. Bowers AR, Meek C, Stewart N. Illumination and reading performance in age-related macular degeneration. Clin Exp Optom. 2001;84:139-47. https://doi.org/10.1111/j.1444-0938.2001.tb04957.x.
74. Fosse P, Valberg A. Lighting needs and lighting comfort during reading with age-related macular degeneration. J Vis Impair Blind. 2004;98:389-409. https://doi.org/10.1177/0145482X0409800702.

75. Asiedu K, Abu SL. The impact of topical intraocular pressure lowering medications on the ocular surface of glaucoma patients: a review. J Curr Ophthalmol. 2019;31:8-15. http://www. sciencedirect.com/science/article/pii/S2452232518301008.

76. Kanthan GL, Wang JJ, Rochtchina E, Mitchell P. Use of antihypertensive medications and topical beta-blockers and the longterm incidence of cataract and cataract surgery. Br J Ophthalmol. 2009;93:1210 LP-1214. http://bjo.bmj.com/content/93/9/1210. abstract.

77. Kirwan JF, Lockwood AJ, Shah P, Macleod A, Broadway DC, King AJ, et al. Trabeculectomy in the 21st Century: a multicenter analysis. Ophthalmol. 2013;120:2532-9. https://doi.org/10.1016/j. ophtha.2013.07.049.

78. Purpura K, Kaplan E, Shapley RM. Background light and the contrast gain of primate $\mathrm{P}$ and $\mathrm{M}$ retinal ganglion cells. Proc Natl Acad Sci. 1988;85:4534-7.

79. Quigley HA, Dunkelberger GR, Green WR. Retinal ganglion cell atrophy correlated with automated perimetry in human eyes with glaucoma. Am J Ophthalmol. 1989;107:453-64.

80. Swanson WH, Sun H, Lee BB, Cao D. Responses of primate retinal ganglion cells to perimetric stimuli. Invest Ophthalmol Vis Sci. 2011;52:764-71.

81. McKendrick AM, Badcock DR, Morgan WH. Psychophysical measurement of neural adaptation abnormalities in magnocellular and parvocellular pathways in glaucoma. Invest Ophthalmol Vis Sci. 2004;45:1846-53.

82. Mutlukan E. A comparison of automated static dark stimuli with the Humphrey STATPAC program in glaucomatous visual field loss. Br J Ophthalmol. 1994;78:175-84.

83. van den Berg TJTP, René van Rijn LJ, Kaper-Bongers R, Vonhoff DJ, Völker-Dieben HJ, Grabner G. et al. Disability glare in the aging eye. Assessment and impact on driving. J Optom. 2009;2:112-8.

84. Green AJ. Visual evoked potentials, electroretinography, and other diagnostic approaches to the visual system. In: Aminoff's electrodiagnosis in clinical neurology. Elsevier; 2012. p. 477-503.

85. Derby GS, Waite JH, Kirk EB. Further studies on the light sense in early glaucoma. Trans Am Ophthalmol Soc. 1926; 24:92-104.

86. Marlow SB. Field of vision in chronic glaucoma; a comparison of fields with full and with reduced illumination. Arch Ophthalmol. 1947;38:43-56. http://europepmc.org/abstract/MED/20258236.

87. Ramulu P. Glaucoma and disability: which tasks are affected, and at what stage of disease? Curr Opin Ophthalmol. 2009;20:92-8. https://www.ncbi.nlm.nih.gov/pubmed/19240541.

88. Medeiros FA, Weinreb RN, Boer R, Rosen E, Driving PN. simulation as a performance-based test of visual impairment in glaucoma. J Glaucoma. 2012;21:221-7. https://www.ncbi.nlm. nih.gov/pubmed/21467952.

89. Nassiri N, Mehravaran S, Nouri-Mahdavi K, Coleman AL. National eye institute visual function questionnaire: usefulness in glaucoma. Optom Vis Sci. 2013;90. https://journals.lww.com/ optvissci/Fulltext/2013/08000/National_Eye_Institute_Visual_ Function.5.aspx.

90. Wang Y, Alnwisi S, Ke M. The impact of mild, moderate, and severe visual field loss in glaucoma on patients' quality of life measured via the Glaucoma Quality of Life-15 Questionnaire: A meta-analysis. Medcine. 2017;96:e8019-e8019. https://www. ncbi.nlm.nih.gov/pubmed/29310323.

91. Owsley C, Ghate D, Kedar S. Vision and Aging. In: Wiley Handbook on the Aging Mind and Brain. 2018. p. 296-314. 